\title{
EL PROYECTO COSTA ESCONDIDA: ARQUEOLOGÍA Y COMPROMISO COMUNITARIO A LO LARGO DE LA COSTA NORTE DE QUINTANA ROO, MÉXICO*
}

\author{
THE COSTA ESCONDIDA PROJECT: ARCHAEOLOGY AND COMMUNITY \\ ENGAGEMENT ALONG QUINTANA ROO'S NORTH COAST, MEXICO
}

\author{
Jeffrey B. Glover ${ }^{1}$, Dominique Rissolo ${ }^{2}$, Jennifer P. Mathews ${ }^{3}$ y Carrie A. Furman ${ }^{4}$
}

\begin{abstract}
El Proyecto Costa Escondida iniciado en 2006 se diseñó para investigar las culturas marítimas previas y posteriores al contacto español, así como para estudiar el paisaje del norte de Quintana Roo, Península de Yucatán, México. Este proyecto no promueve una agenda de desarrollo "tradicional", sino que se inserta en la crítica del desarrollo para ampliar los límites del compromiso comunitario a través del aprendizaje social. Al encontrarse lejos de los principales sitios turísticos de la costa del Caribe, el área cuenta con una industria de turismo en expansión asociada a la Isla Holbox. En este artículo se discuten las experiencias de este proyecto arqueológico que integra a varios actores de la región y, más importante aún, traza una estrategia de investigación transparente que involucra activamente a estos individuos a medida que el proyecto avance.
\end{abstract}

Palabras claves: arqueología maya, arqueología comunitaria, turismo, aprendizaje social.

We established the Costa Escondida Project in 2006 to investigate the pre-contact and historic maritime cultures and landscape of northern Quintana Roo, Mexico. While removed from the major tourist centers of the Caribbean coast, this area has a burgeoning ecotourism industry associated with Isla Holbox. This paper will discuss our experiences as an archaeologically focused project working with the various stakeholders in the region, and more importantly, outline a research strategy that is transparent and will actively engage these stakeholders as the project progresses.

Key words: Maya archaeology, community archaeology, tourism, social learning.

Los arqueólogos realizan el trabajo de campo de manera muy distinta a los etnógrafos, pues a menudo vivimos en las áreas de estudio como residentes temporales de las comunidades locales. Nuestras prioridades están enfocadas a cumplir con los objetivos de la temporada y no en las implicaciones de nuestra presencia como residentes foráneos en estos lugares. Frecuentemente olvidamos que nos encontramos dentro de una cultura contemporánea y que nuestro estudio del pasado tiene ramificaciones en el presente. Afortunadamente, la arqueología como disciplina se ha esforzado para acotar la brecha entre el registro arqueológico y las personas que claman por éste como parte de su herencia; esto lo evidencian los recursos del Congreso Mundial de Arqueología (WAC por sus siglas en inglés), las sesiones recientes de las juntas profesionales y numerosas publicaciones (p.ej., Ardren 2002; Arthur et al. 2010; Breglia 2006; Castañeda y Mathews 2008; Edgeworth, ed. 2006; Gerunds 2007; Kus 2010; Lynott y Wylie 2000; Marshall 2002; McGuire 2008; Rissolo y Mathews 2005; Sabloff 1998; Santoro et al. 2003; Walker 2009). Sin embargo, mientras los etnógrafos están capacitados para lidiar con el reto de vivir en las comunidades donde trabajan, la mayoría de los arqueólogos no

* Artículo seleccionado del conjunto de ponencias presentadas en el Simposio Shifting from Object-centered Research to People-focused Application: Current Approaches to Public Archaeology from Latin America and the Caribbean, realizado en el marco de la 75 reunión anual de la Sociedad de Arqueología Americana, Saint Louis, Missouri, EUA, abril 2010. Este manuscrito fue evaluado por investigadores externos y editado por Reiko Ishihara-Brito y Cameron Griffith, en su calidad de editores invitados de la Revista.

1 Departamento de Antropología, Georgia State University, P.O. Box 3998, Atlanta, GA 30302-3998, EUA. jglover@gsu.edu

2 Waitt Institute, 5786 La Jolla Blvd., La Jolla, CA 92037, EUA. dominique@waittfoundation.org

3 Departamento de Sociología y Antropología, Trinity University, One Trinity Place, San Antonio, TX 78212-7200, EUA. jmathews@trinity.edu

4 Departamento de Antropología, Georgia State University; Departamento de Ingeniería Biológica y Agrícola, University of Georgia, Driftmier Engineering Center, Athens, GA 30602-4435, EUA. cfurman@uga.edu 
lo está. Debemos reconocer estas carencias e intentar tratarlas como parte, y no aparte, de nuestros proyectos de investigación arqueológica.

En este artículo discutimos las maneras como nuestro trabajo podría promover otros proyectos mayores entre quienes viven y trabajan en una región. Examinamos primero varios enfoques sobre arqueología y compromiso comunitario y esbozamos uno nuevo basado en el aprendizaje social y la creación de una comunidad de práctica. Después exponemos los antecedentes del Proyecto Costa Escondida (PCE), los actores de la región que lo conforman y las formas de compromiso empleadas hasta la fecha. También sondeamos los retos que podrían presentarse en el intento de desplazarse desde una arqueología con base en los objetos a una con base en los agentes.

\section{Acercamientos a la Arqueología de Compromiso Comunitario}

En la literatura sobre arqueología de compromiso comunitario se vislumbran dos tipos generales de acercamiento para abordar este tipo de investigaciones (para una caracterización diferente ver Moser et al. 2002:222). Uno de ellos se conforma de etnografía sobre arqueólogos y proyectos arqueológicos (Breglia 2006; Castañeda y Matthews 2008; Edgeworth, ed. 2006) y otro está más dirigido al desarrollo participativo, nombrado bajo la categoría de "proyectos comunitarios aplicados". Debemos señalar que estos enfoques no son excluyentes y que continuamente se intercalan. Finalmente, presentamos el concepto de aprendizaje social, como un proceso que aborda ciertas críticas a los proyectos comunitarios, lo cual podría complementar los estudios etnográficos sobre arqueología.

\section{Etnografía sobre la práctica arqueológica y proyectos comunitarios aplicados}

La investigación etnográfica sobre las implicaciones que tienen los proyectos arqueológicos en la identidad local y en las políticas de patrimonio ha sido un campo activo de estudio en la última década (Breglia 2006; Castañeda y Matthews 2008; Edgeworth, ed. 2006; Mortensen y Hollowell 2009). Distinguimos dicho enfoque porque estas etnografías de orientación más teórica no siempre involucran a la población local como lo hacen los proyectos aplicados; por el contrario, la comunidad local tiende a volverse otro objeto de estudio junto con los arqueólogos (Edgeworth 2006:15).

La meta de los proyectos aplicados de arqueología comunitaria es involucrar activamente a la comunidad local, generalmente descendiente del lugar, en el proceso arqueológico (p.ej., Brumfiel 2000; Marshall 2002). Moser et al. (2002:229) nombran siete aspectos que los proyectos aplicados comunitarios deben tratar de incluir. También advierten que los arqueólogos deben esforzarse activamente por asegurar que los proyectos no se condicionen por sus agendas. Sin estos esfuerzos, la comunidad se convierte en nada más que un recipiente pasivo de información (Moser et al. 2002:223).

Este tipo de proyectos se enfoca a estudiar las necesidades, intereses y capacidades de la comunidad como parte del proceso hacia un desarrollo sostenible. Mientras que en el pasado estos trabajos hicieron estricto hincapié en las necesidades comunitarias, recientemente algunos investigadores (p.ej., Dilling y Lemos 2011; Li 2007; Kretzmann y McKnight 1993) han señalado que dicho enfoque no empodera a la población local, sino que, por el contrario, crea una "comunidad clientelar" que podría agudizar o reproducir las desigualdades (Kretzmann y McKnight 1993:5). Kretzmann y McKnight (1993:5) proponen cambiar la orientación hacia un "desarrollo sustentado en la capacidad" que incorpore a organizaciones y asociaciones locales. Argumentan que esta perspectiva empodera a los miembros de la comunidad y aprovecha la fuerza estructural de las relaciones comunitarias ya existentes. La literatura en torno a la "sistematización" también cae dentro de esta categoría. "La sistematización es un proceso de reconstrucción y reflexión analítica sobre una experiencia vivida. Implica un proceso de investigación, análisis y documentación" (GTZ 2011:2). Este enfoque provee un marco teórico técnico para ayudar a la gente que trabaja en planes y programas de desarrollo comunitario. Como evaluación se concentra en la documentación de, y compromiso con, organizaciones existentes asociadas con el plan de desarrollo y brinda métodos explícitos para trazar el avance del proyecto. Aunque es útil como forma de valoración, no altera las cuestiones subyacentes asociadas al desarrollo participativo; cabe mencionar que además muchos de los actores locales no se contemplan en la formulación del proyecto.

Lo anterior también es un problema para la arqueología de compromiso comunitario puesto que frecuentemente pasa por alto la continuidad 
de las relaciones patrón-cliente. Este es un modelo que culpablemente hemos promovido, al establecer en esencia relaciones superficiales donde nos colocamos en la posición de expertos y, por extensión, desalentamos la participación de la comunidad en el procesos de investigación. Para contrarrestar esta tendencia, necesitamos repensar las maneras en que nuestro estudio pueda ser útil a los miembros de la localidad. Dilling y Lemos (2011) discuten el proceso de crear una "ciencia utilizable". Proponen que los científicos y usuarios de la ciencia generalmente perciben las necesidades de manera distinta y que es importante "navegar y acotar cualquier diferencia que pueda existir entre lo que los científicos creen que es útil y lo que en la práctica realmente lo es... [al] establecer una visión compartida" (Dilling y Lemos 2011:681 [traducido por los autores]) de lo que es conocimiento útil (ver también Berkes 2009). Una forma de hacer esto es desarrollar una comunidad de práctica a través del proceso de aprendizaje social.

\section{Aprendizaje social}

Un tercer camino hacia el desarrollo de un proyecto arqueológico de efectivo compromiso comunitario se encuentra en el aprendizaje social y la creación de una comunidad de práctica. Como Wylie (1995:267) menciona, hacer disponible la información arqueológica para la localidad no es suficiente, porque ni empodera a las comunidades locales ni las hace partícipes activas en los procesos de investigación arqueológica (sentimiento compartido por Moser et al. 2002).

En un contexto de aprendizaje social, los individuos desarrollan "nuevas relaciones que construyen sobre las ya existentes y transforman las que son adversas a medida que aprenden acerca de la honradez de los demás y a apreciar la legitimidad del punto de vista de cada quien" (Reed 2008:2420 [traducido por los autores]). El aprendizaje social es en primer lugar un proceso y por lo tanto no está sujeto a encontrar maneras de facilitar la entrega de un producto predeterminado, crítica lanzada frecuentemente a los proyectos de desarrollo participativo. El objetivo es crear una atmósfera en donde todas las partes interesadas aprendan una de la otra, desarrollen relaciones interdependientes y combinen conocimiento y experiencia con personas de distintos contextos para tratar cuestiones o necesidades específicas (Cox 2005; Hildreth y Kimble
2004; Leeuwis y Pyburn 2002). Las soluciones innovadoras a los problemas están conformadas por las relaciones, el diálogo abierto y la acumulación de observaciones compartidas (Tekelenburg et al. 2002). Este enfoque crea una comunidad de práctica (p.ej., Lave y Wenger 1991; para una crítica del uso del concepto, ver Cox 2005), que en este caso se aboca a asuntos arqueológicos. En este sentido, una comunidad de práctica se define como:

un conjunto de personas que aúnan esfuerzos alrededor de compromisos mutuos en algún empeño. Maneras de hacer las cosas, maneras de hablar, creencias, valores, relaciones de poder, en pocas palabras, la emergencia de prácticas en el curso de este esfuerzo mutuo. Como constructo social, una comunidad de práctica es distinta de la comunidad tradicional, primeramente porque está definida simultáneamente por sus miembros y por la práctica en la que los miembros se involucren. (Esto no significa que las comunidades de práctica sean necesariamente igualitarias o consensuales, sino que simplemente su afiliación y prácticas emergen de un compromiso mutuo). [Eckert y McConnel-Ginet 1992:464 (traducido por los autores)].

La formación de una comunidad de práctica es un proceso interactivo y ayuda a transportar el flujo de conocimiento de un carácter lineal y jerárquico a uno multidireccional. Esto es muy necesario si buscamos romper viejos patrones de diseminación del saber. En nuestro caso, vislumbramos un proceso que construya una comunidad de práctica formada en asociación con los asuntos de manejo de patrimonio y que promueva no sólo nuestros intereses, sino los de todos los actores de la región.

\section{Antecedentes del Proyecto}

El Proyecto Costa Escondida comenzó en 2006 bajo la dirección de Rissolo y Glover. Nuestra meta de investigación es comprender mejor cómo los mayas de la costa norte de Quintana Roo, Península de Yucatán (Figura 1), se adaptaron a este medio ambiente a lo largo de milenios y cómo estaban ligados a rutas comerciales mayores alrededor de la península y a distintas esferas de interacción. Nuestros intereses de investigación se extienden 
desde el Preclásico Medio (ca. 700 a.C.) a la época histórica (Glover et al. 2011). Mathews dirige la parte histórica del estudio, centrándose en varias de las industrias extractivas (palo de tinte, caña de azúcar) que operaron a lo largo de la costa en el siglo XVIII y principios del siglo XX (Figura 1).

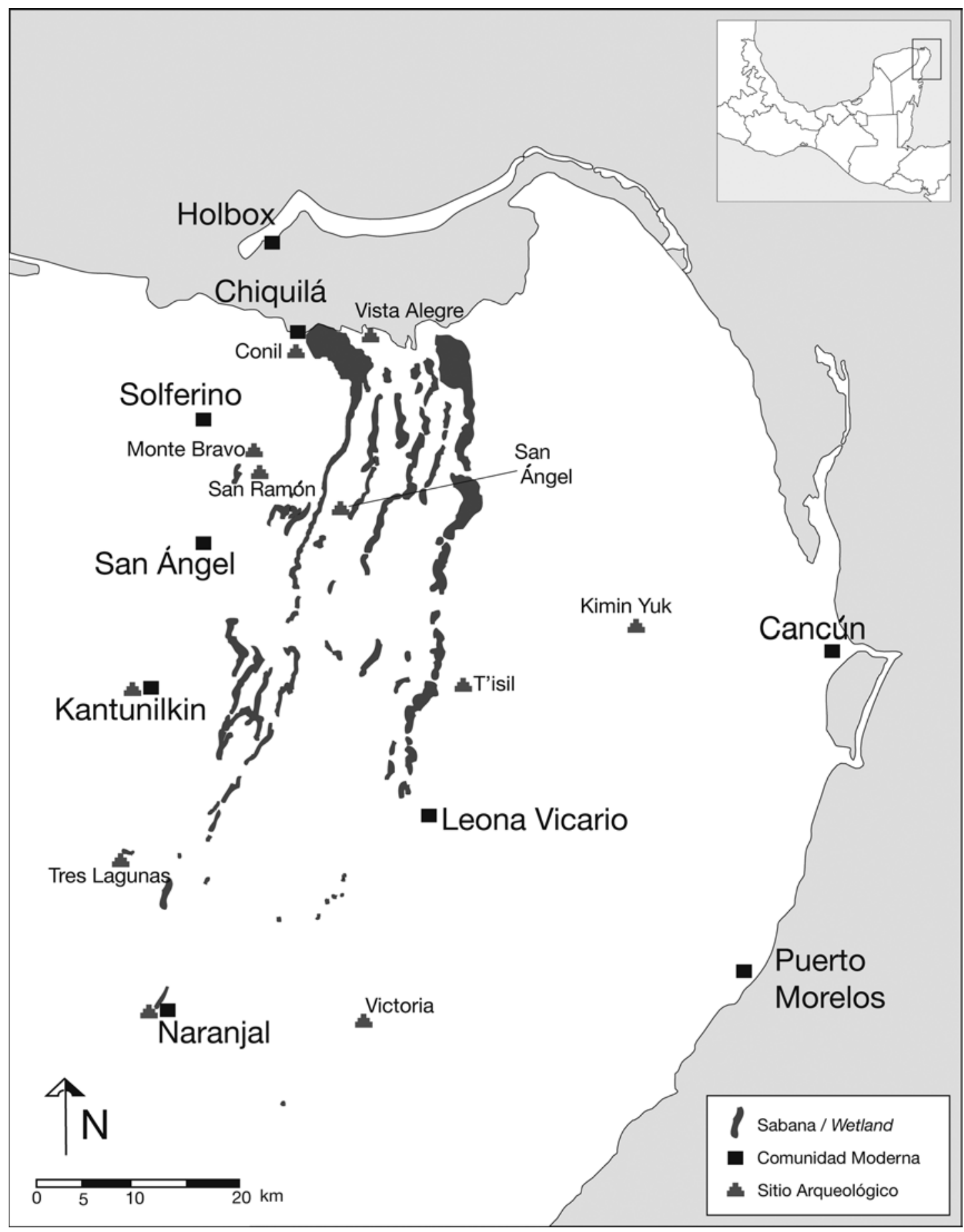

Figura 1. Mapa de la Región de Yalahau con los lugares mencionados en el texto. Map of the Yalahau Region with places mentioned in text. 
Un reto, al trabajar en esta región, es su extrema atención hacia el turismo, particularmente por su relación con las ruinas arqueológicas. El Estado de Quintana Roo (hogar de la Riviera Maya) está dominado por una creciente industria turística, por lo que no puede ser ignorada. Consecuentemente, debemos involucrarnos con los pros y contras; aunque no estamos buscando promoverla dentro de las comunidades con las que trabajamos, su desarrollo está sucediendo estemos o no implicados, como es evidenciado por los nuevos anuncios de publicidad de la Isla Holbox (Figura 2).

A pesar de que el turismo se encuentra todavía en un estado inicial en la isla, hemos analizado otros desarrollos en la región para predecir la trayectoria potencial del mismo. Éstos van desde parques privados llamados "ecoturísticos", a empresas más tradicionales de tipo corporativo a gran escala. Experiencias culturales alternativas y más sustanciales están disponibles para los visitantes de la Península de Yucatán e incluyen sitios arqueológicos y museos manejados por el Instituto Nacional de Antropología e Historia (INAH), reservas de la biósfera, turismo de pequeña escala con base en el ejido y otros esfuerzos de desarrollo. Una amplia gama de agentes está actualmente activa en nuestra región de estudio y será descrita en la siguiente sección.

\section{Actores en la Región de Estudio}

Debido a la diversidad de actores dentro de la zona, sus proyectos, intereses y agendas abarcan desde los que se enfocan estrictamente a su comunidad, hasta aquellos que buscan unir a múltiples poblaciones. Algunos tienen un vasto interés en el patrimonio cultural, mientras que otros no tienen un vínculo claro con éste, por lo que apoyan menos los planes para el turismo y por ende la investigación arqueológica. Dada esta dicotomía, requerimos ser precavidos, reflexivos y humildes acerca de cómo proceder con nuestro proyecto.

\section{Ejido de San Ángel/Chiquilá}

El primero de los actores locales a tratar es la comunidad de Chiquilá, la cual es la puerta de entrada a la Isla Holbox y la comunidad con

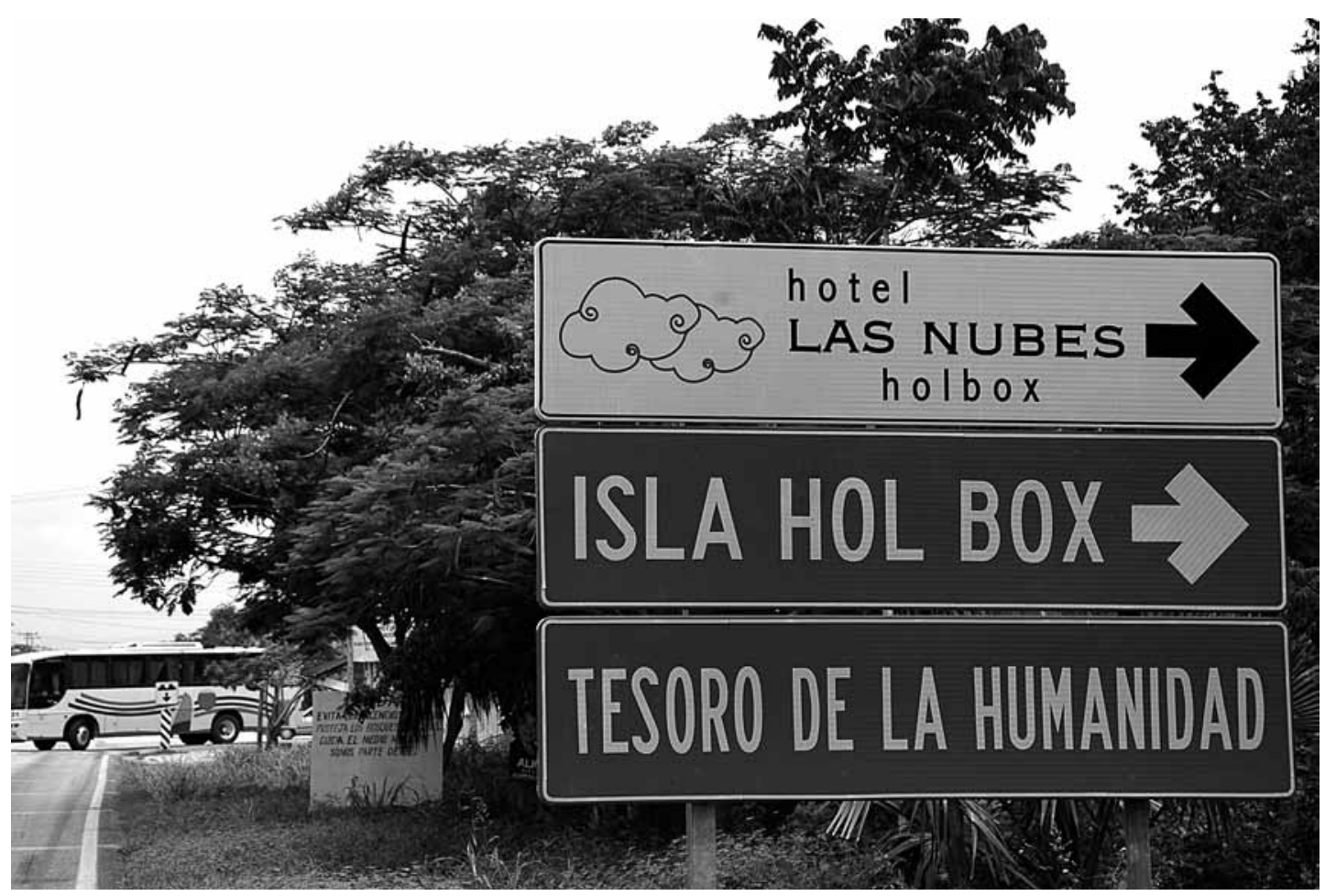

Figura 2. Anuncio publicitario reciente de la Isla Holbox.

Recent sign advertising Isla Holbox. 
la que estamos más estrechamente involucrados (Figura 1). Chiquilá es un anexo del ejido de San Ángel y presenta una mezcla de descendientes tradicionales y no tradicionales. Al final de la década de los setenta o principios de los ochenta, un grupo de veracruzanos migró a este lugar, lo que parece haber desplazado a mucha de la población indígena maya. Debido a que los nuevos miembros de la comunidad eran "foráneos", probablemente se aliaron con la población maya de San Ángel al interior. La población no maya de Chiquilá presenta retos adicionales debido a su historia y a la ausencia de una conexión cultural directa con los sitios arqueológicos locales, aunque tal conexión tampoco asegura que la arqueología sea vista como relevante (ver Breglia 2006).

\section{Ejido de Solferino}

Solferino es la siguiente comunidad al sur de Chiquilá (Figura 1) con una larga historia en el área (Gust y Mathews 2011; Sullivan 2004). Glover trabajó en Solferino durante su proyecto de tesis y llegó a conocer a muchos de los miembros de la comunidad. Ésta ha sido la única comunidad en donde se ha tenido que firmar un convenio elaborado en conjunto con los miembros del ejido, el cual definía la naturaleza de la investigación arqueológica que sería realizada. Esto indicaba el interés de la comunidad de estar más involucrada en el proceso arqueológico, así como en mantener cierto control sobre lo que sucediera con el conocimiento generado.

\section{Puerta Verde}

La organización Puerta Verde, fundada en 1996, ha reunido a siete comunidades (incluidas Solferino, Chiquilá, San Ángel y Holbox), doce grupos organizados y 250 beneficiarios directos (individuos que podrían obtener beneficios del turismo) entre Cobá y la Isla de Holbox (Puerta Verde 2010). Las metas de esta cooperativa son promover un turismo sostenible que ayude a proteger los recursos naturales y culturales del área (Figuras 3 y 4). El grupo ha recibido apoyo externo de la Asociación Civil Kanché. que tiene sede en Cancún (Kanché 2010), la cual promueve fuertemente la iniciativa de Puerta Verde, tanto en su página web como en Facebook.

\section{Turismo y cooperativas en Chiquilá}

Hay varias cooperativas turísticas en Chiquilá que están afiliadas a Puerta Verde, sin embargo, ninguna está vinculada a los sitios arqueológicos circundantes. Ellas generan ganancias al llevar turistas de Chiquilá u Holbox, para avistar aves y nadar con el tiburón ballena más grande del mundo (World Wildlife Fund 2010). Estas aguas están salvaguardadas al formar parte del área protegida Yum Balam, otro actor en la región.

\section{Yum Balam}

Establecida en 1994 por la Comisión Nacional de Áreas Naturales Protegidas (CONANP), Yum Balam es un área protegida de animales y plantas,
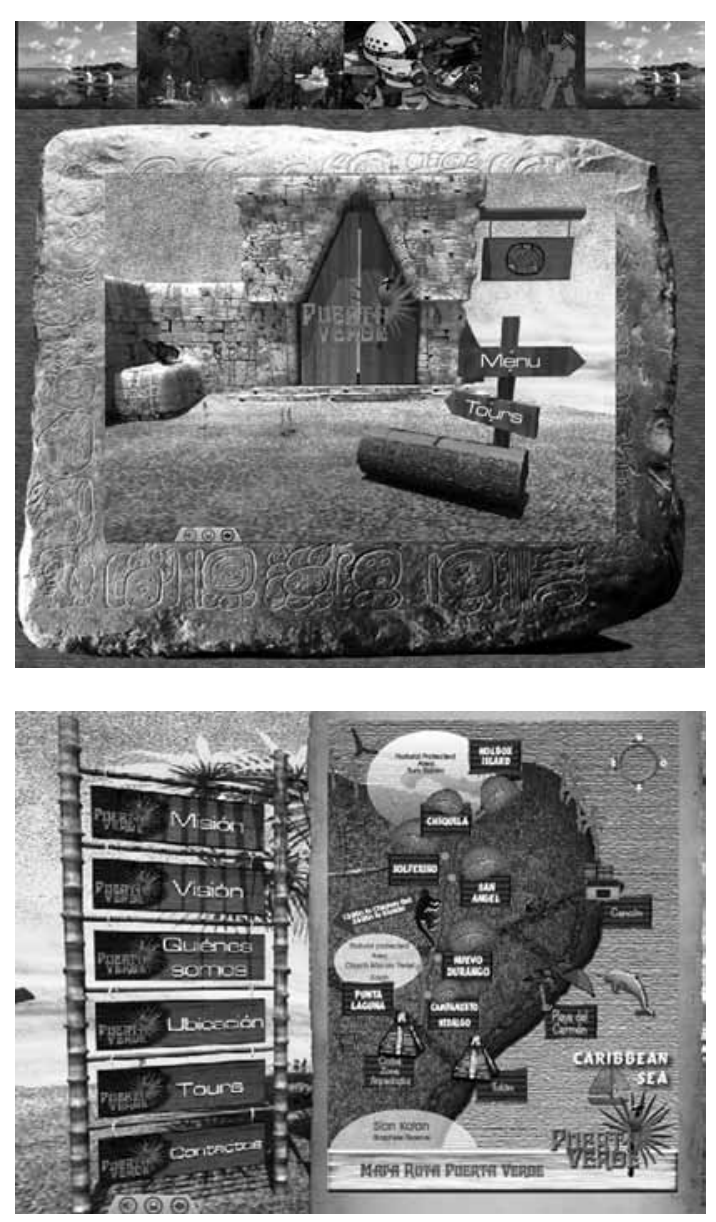

Figura 3. Captura de la pantalla de la página web antiguo de Puerta Verde.

Screen shot of former Puerta Verde webpage. 


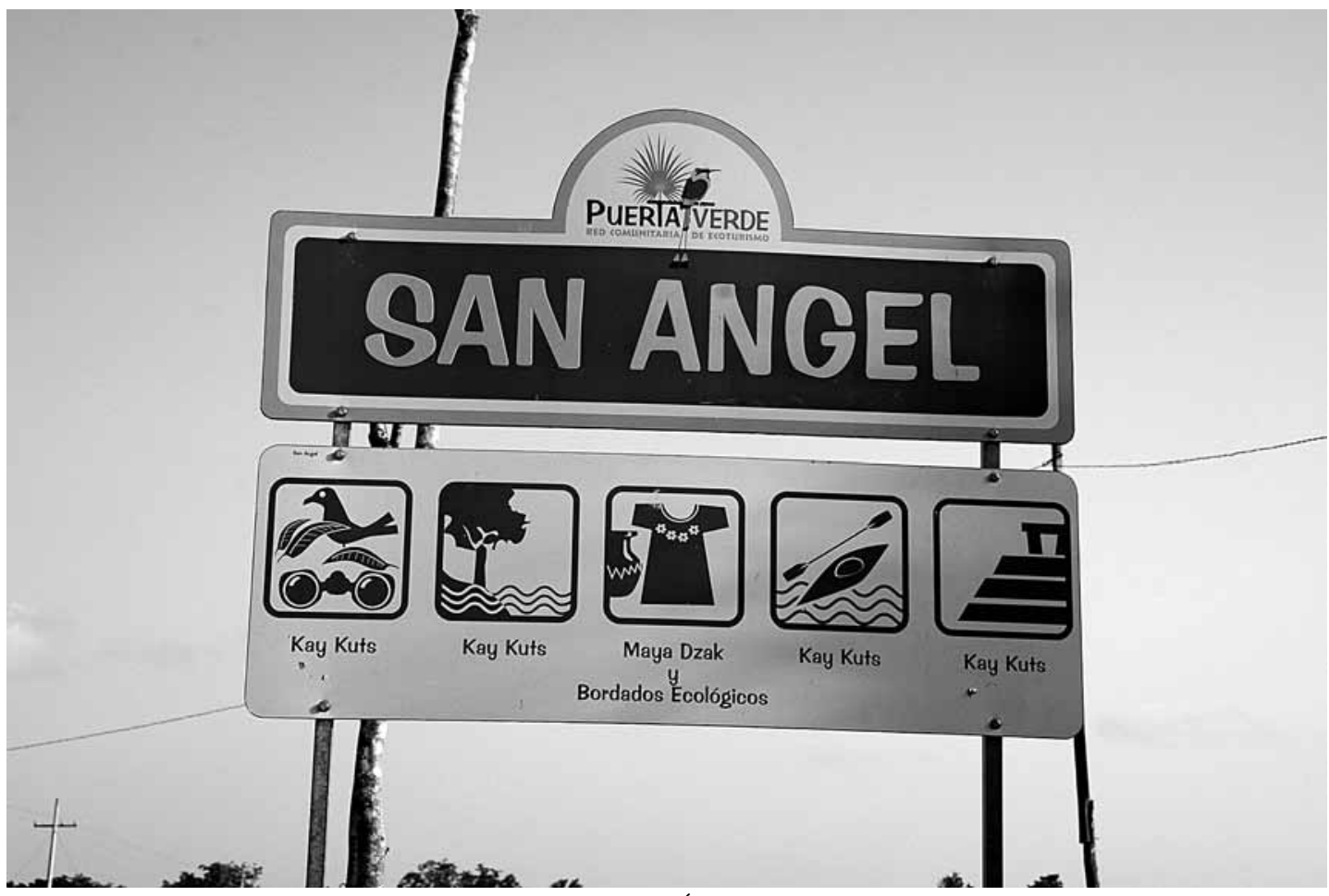

Figura 4. Anuncio de Puerta Verde fuera de la comunidad de San Ángel.

Puerta Verde sign outside of the community of San Ángel.

que forma parte de la porción norte del protegido Arrecife Mesoamericano (Yum Balam 2010). La oficina principal de esta reserva se ubica en Cancún pero tiene un par de botes patrulla y representantes locales en Chiquilá y Solferino. Mientras que la reserva fue importante para fundar Puerta Verde y es promovida en su página web, Yum Balam parece tener escasa infraestructura turística y nada de turismo relacionado con los sitios arqueológicos de la región. Sin embargo, está estrechamente involucrada en el estudio y protección del tiburón ballena del Atlántico mexicano, lo cual podría suscitar el desarrollo de dicha infraestructura a medida que la Isla Holbox crezca y los intereses de proyectos de desarrollo comunitario, a escala regional, se incrementen.

\section{Instituto Nacional de Antropología e Historia}

El INAH es un actor fundamental respecto al patrimonio cultural (o manejo del legado). Los restos arqueológicos son considerados en México patrimonio nacional y pertenecen al Estado; por lo tanto, cualquier proyecto que implique recursos arqueológicos involucra a este Instituto de alguna manera, tanto a nivel estatal como federal.

\section{Proyecto Costa Escondida}

Nos percibimos como un actor más de la región. Como se discutió antes y aunque nuestros proyectos de investigación han sido de temporadas cortas, hemos trabajado a lo largo de la costa norte de manera formal desde 2006. Las implicaciones de la naturaleza de nuestro estudio y la importancia de considerarnos parte del grupo de actores, serán cuestiones expuestas a continuación.

\section{Relaciones Anteriores con los Actores}

Comprender cómo hemos involucrado a la comunidad local ayudará a evidenciar los obstáculos que tendremos que enfrentar a medida que avancemos e intentemos emplear un enfoque basado en el aprendizaje social. En el pasado hemos realizado esfuerzos para hacer nuestro trabajo lo más accesible y transparente posible; esto es ciertamente un paso clave para involucrar a los 
miembros comunitarios y abre las oportunidades para crear una comunidad de práctica. Aunque ésta no es una idea revolucionaria, en términos prácticos es una tarea exigente en lugares como Vista Alegre y Xuxub. Estos sitios arqueológicos se localizan en una remota isla interior de la costa y sólo son accesibles por barco. Debido a esto, más allá de los trabajadores que participan con nosotros, no tenemos supervisión de la comunidad y no podemos involucrarnos con personas que en otras circunstancias podrían hacer visitas casuales al proyecto. Los trabajadores con quienes hemos generado una relación nos han contado acerca de los rumores que circulaban en Chiquilá alrededor de nuestro "misterioso" trabajo en la isla. Una vez que ellos reportaron a la comunidad lo tedioso del proceso arqueológico e informaron sobre cómo éste era la antítesis del saqueo, los rumores finalmente pararon. Nos atrevemos a decir que esta situación es un asunto común en proyectos arqueológicos en cualquier lugar.

Otro método que hemos empleado para involucrar a los agentes de la región es a través de la diseminación de nuestros hallazgos de investigación. Hemos presentado esta información en un sinfín de diferentes formatos. Siempre llevamos a las comunidades locales copias del permiso que nos expide el INAH, nuestra propuesta de trabajo en español y compartimos informes del INAH recientes y cualquier otra publicación pertinente. Glover y Mathews también han trabajado en la producción de carteles para cada comunidad. Los educadores locales emplearon el cartel de Mathews para enseñar la historia reciente del área a los niños del lugar. Por su parte, los miembros del ejido de Solferino se mostraron entusiasmados de tener algo que compartir con los turistas que lleguen a la comunidad. En suma, se discutió cómo esta información podría ser utilizada para proteger estos lugares, pues algunos individuos de la comunidad han vendido sus parcelas. A pesar de estos beneficios, la difusión de los carteles ha sido una tarea extremadamente ardua. Aunque nos hacemos responsables de ella, las demandas de trabajos académicos, particularmente las presiones en torno a publicaciones para promoción y titularidad, proveen una recompensa mínima para este tipo de trabajo. Como se mencionó anteriormente, éste es otro ejemplo de trasferencia unilateral de información y no fomenta realmente un intercambio más dialéctico de conocimiento.

\section{Discusión}

Un aspecto importante del concepto de comunidad de práctica con el que empatizamos el define a los arqueólogos como parte de la "comunidad", y da un nuevo significado a la idea de arqueología de compromiso comunitario a medida que nos involucra como un agente más en el proceso. El aprendizaje social es también una novedad en la manera en que los proyectos de desarrollo participativo son frecuentemente llevados a la práctica cuando tienden a ser conducidos por la agenda de un grupo específico (p.ej., Ferguson 1994). A través del empleo del enfoque de aprendizaje social, el cual requiere un mediador externo y la creación de herramientas de integración, esperamos nivelar el terreno de juego y evitar privilegiar la perspectiva o agenda particular de un grupo sobre otro.

Una situación que nos empujó hacia el aprendizaje social y a la creación de una comunidad de participación fue el continuo navegar dentro y entre estos varios actores con los que trabajamos. Aunque tenemos un entendimiento básico de estos grupos y de cómo interactúan, no tenemos la profundidad de comprensión que se obtiene a través de una investigación etnográfica prolongada. Nos hemos esforzado constantemente en tratar de comprender a estos sectores únicamente sobre la marcha, producto de fondos limitados y la realidad de un calendario académico que restringe nuestro tiempo de campo a las vacaciones de invierno y verano. Como resultado, tenemos que volver a entablar nuestras conexiones cada año y ajustar nuestros procedimientos de acuerdo al surgimiento de nuevos desarrollos comunitarios.

Mientras que el aprendizaje social y el desarrollo de una comunidad de práctica son buenas ideas, reconocemos que este proceso no se desarrollará orgánicamente. La principal barrera será saber si todos los actores están lo suficientemente interesados o no en el patrimonio cultural de la región como para participar. Admitimos que debemos iniciar este proceso. En segundo lugar, estamos conscientes de que aquellos grupos en el poder, particularmente las agencias federales, pueden no querer atender las inquietudes e ideas de los agentes no federales. El INAH tiene jurisdicción esencialmente sobre todo el patrimonio cultural del país y generalmente ocupa este poder para tomar decisiones unilaterales sin consultar a la comunidad. Esto fue recientemente evidenciado cuando el Instituto juzgó importante 
proteger el monumento de la serpiente emplumada que documentamos en Vista Alegre; aunque completamente legal y llevado a cabo para proteger dicho monumento labrado, su traslado a una instancia del INAH en Chetumal (a seis horas de distancia en vehículo) no fue visto de manera favorable por la comunidad, incluso cuando dicho Instituto ofreció crear una réplica.

Éste no es ciertamente el único punto de tensión dentro y entre los varios actores asociados a nuestro proyecto. También es importante reconocer que nos encontramos en una posición de poder, a pesar de que lo queramos o no. Nuestra riqueza relativa, el estatus como extranjeros y los títulos académicos, no son elementos que se puedan ignorar, sino que pueden ser intimidantes e incluso amenazadores para otros actores. ¿Puede un ambiente basado en el aprendizaje social neutralizar estas relaciones de poder preexistentes? Aunque no sabemos la respuesta, sabemos que vale la pena intentarlo.
Puesto que buscamos fondos para apoyar esta tarea, existen pasos iniciales que podemos comenzar a realizar. Uno es darnos el tiempo para reunirnos con los representantes de todos los grupos actores del área (Figura 5). A medida que iniciamos un trabajo arqueológico más intensivo en la costa norte, debemos priorizar estas relaciones para encontrar qué es lo que ellos esperan obtener de nuestro estudio, limitar los malos entendidos acerca de nuestros objetivos de investigación y contemplar las necesidades de estos actores como una manera de ayudarnos a planear futuros proyectos. Otro paso a seguir es el desarrollo de una página web bilingüe (español e inglés) del PCE que incluya toda nuestra información de contacto, antecedentes acerca del proyecto de investigación y acceso a nuestros textos y reportes. Involucrar al sector educacional es otro camino que nos gustaría recorrer. Puede ser que lo anterior no abarque al total de los actores de manera colectiva pero cada caso puede tratarse individualmente para integrar a los grupos interesados.

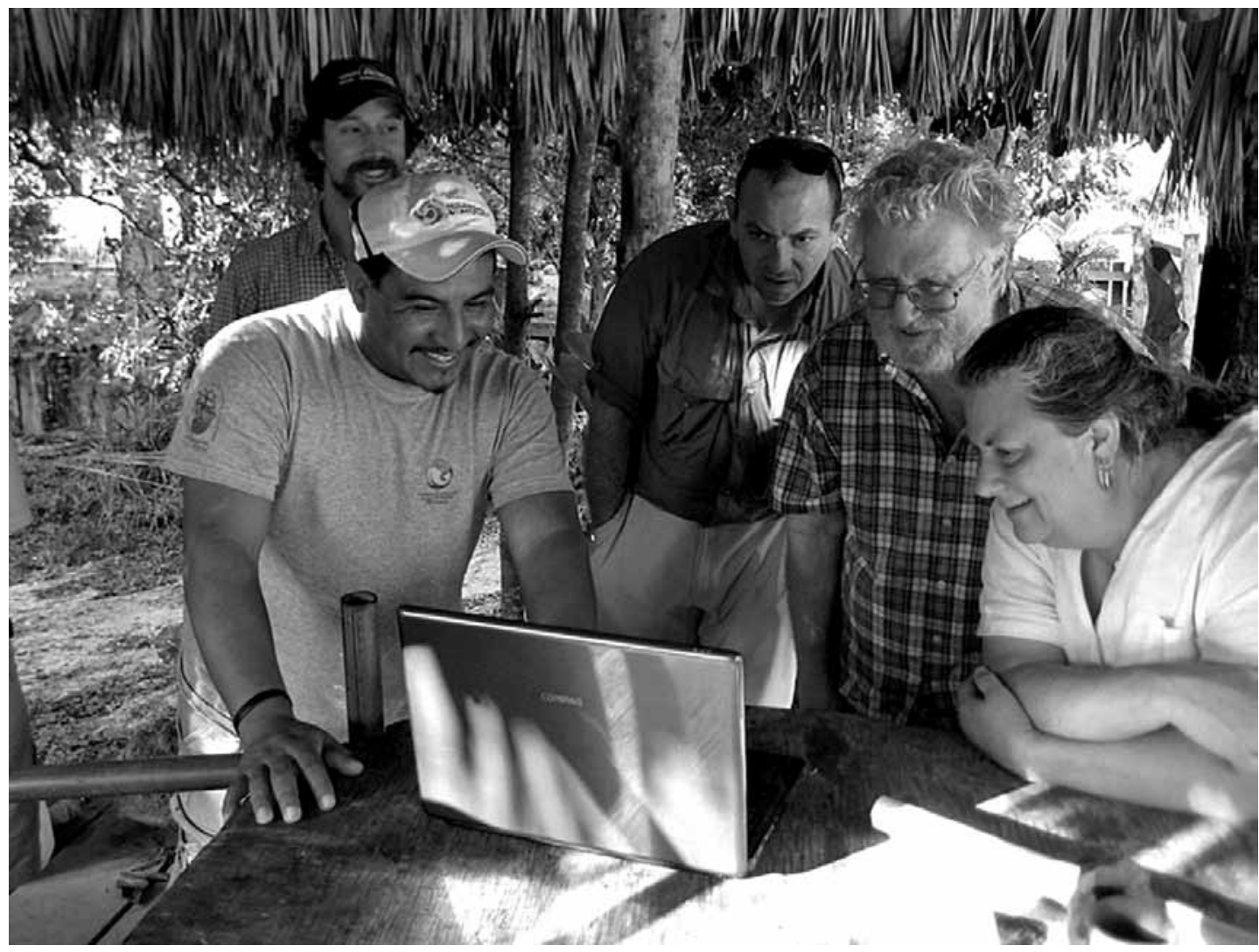

Figura 5. Foto de una cita con miembros de Puerta Verde y el PCE.

Photo of a meeting between members of Puerta Verde and the PCE. 
Otra opción complementaria en el desarrollo de una comunidad de práctica es la que Castañeda (2008) ha propuesto. Su idea es contar con un etnógrafo que trabaje con nosotros, registrando en detalle los contextos sociales e históricos del proyecto arqueológico. Esta descripción "densa" sería parte integral del registro arqueológico; además, nos gustaría impulsar a otros etnógrafos a trabajar con varios de los actores. Aunque reconocemos plenamente los beneficios del trabajo etnográfico realizado en conjunto con un proyecto arqueológico (ver McGuire 2008:145), debe quedar claro que el etnógrafo sería entonces otro actor que debe ser tratado y concebido como tal.

\section{Comentarios Finales}

Este proyecto no promueve una agenda de desarrollo "tradicional", sino que se inserta en la crítica del desarrollo para ampliar los límites del compromiso comunitario a través del aprendizaje social. Nos damos cuenta, que nuestros enfoques hacia el compromiso comunitario han estado lejos de considerarse adecuados y todavía se encuentran en desarrollo; tenemos el interés de formalizar nuestras relaciones con los demás actores a través de un enfoque basado en los beneficios del aprendizaje social y en la creación de una comunidad de práctica. A lo largo de estos años hemos forjado una estrecha relación con la población del área y sentimos que este acercamiento nos permitirá colaborar en el desarrollo sustentable de bases en el área.

No obstante, sin un plan adecuado nos enfrentamos lentamente a esta tarea, pues estamos conscientes de los riesgos de proceder sin un mejor entendimiento del presente etnográfico. Dado que no comprendemos completamente las relaciones sociopolíticas entre los actores y requerimos también de un moderador en un lugar que nos reúna a todos, debemos ser cuidadosos de las consecuencias no planeadas de nuestras acciones. Sabemos que la práctica arqueológica juega un rol en la construcción de la identidad en el presente (p.ej., Breglia 2006; Hutson 2010) y que no queremos alimentar inadvertidamente las tensiones dentro y entre los grupos agentes.
$\mathrm{Al}$ concluir nuestro proyecto de investigación esperamos escribir un libro con nuestras historias acerca de las dinámicas relaciones humanas que se han desplegado a través de los milenios desde que los recolectores agricultores de la costa que empleaban cerámica establecieron por primera vez sus asentamientos en la costa norte. No pretendemos terminar estas historias al tiempo del contacto español o a principios del siglo XX. Un entendimiento detallado de la vida actual en esta área no debiera ser sólo una nota al pie o un apéndice, sino parte integral de este libro. A través de la creación de una comunidad de práctica y de la generación de nuevo conocimiento, esperamos crear un proyecto colaborativo que nos permita realmente desplazarnos de una arqueología basada en objetos a una basada en agentes.

Agradecimientos: Agradecemos a Reiko Ishihara-Brito y a Cameron Griffith por la invitación a participar en esta edición especial de Chungara. Nuestra contribución también se vio altamente beneficiada de sus comentarios junto con los de otros dos críticos. Esta investigación no podría haber sido posible sin el apoyo de los fondos provenientes del Research Initiation Grant de Georgia State University, los fondos de Faculty Development Grant de Trinity University y la ayuda generosa del Waitt Institute. Agradecemos al Consejo de Arqueología del INAH por otorgarnos los permisos para realizar esta investigación. También agradecemos a Victoria Beltrán Kuhn por su trabajo de traducción. Nada de esto hubiera sido posible sin la amabilidad y apoyo de las personas de Chiquilá, Solferino y San Ángel. Tampoco hubiéramos podido completar mucho del trabajo sin la ayuda de nuestro equipo local proveniente de Chiquilá: Roberto Echevaría (nuestro capitán), Abel Mendezaba Orduña, Alfonso Martínez Márquez, Feliciano Mukul Yah, Gerardo Mendezaba Orduña y Apolinar Caixba Chagarla. Agradecemos a la gente de Yum Balam por su interés en nuestro proyecto y también a Pepe Quintal por su amistad y entusiasmo en la conservación del valioso patrimonio natural y cultural del norte de Quintana Roo. 


\section{Referencias Citadas}

Ardren, T. 2002. Conversations about the production of archaeological knowledge and community museums at Chunchucmil and Kochol, Yucatán, México. World Archaeology 34:379-400.

Arthur, K.W., J.W. Arthur, M.C. Curtis, B. Lakey, J. LesurBegremariam eY. Ethiopia 2010. Fire on the mountain: dignity and prestige in the history and archaeology of the Borada Highlands in Southern Ethiopia. The SAA Archaeological Record 10(1):17-21.

Berkes, F. 2009. Evolution of co-management: Role of knowledge generation, bridging organizations and social learning. Journal of Environmental Management 90:1692-1702.

Breglia, L. 2006. Monumental Ambivalence: The Politics of Heritage. University of Texas Press, Austin.

Brumfiel, E.M. 2000. Making history in Xaltocan. En Working Together: Native Americans and Archaeologists, editado por K.E. Dongoske, M.S. Aldenderfer y K. Doehner, pp. 181-190. Society for American Archaeology Press, Nueva York.

Castañeda, Q. 2008. The "ethnographic turn" in archaeology: research positioning and reflexivity in ethnographic archaeologies. En Ethnographic Archaeologies: Reflections on Stakeholders and Archaeological Practices, editado por Q.E. Castañeda y C.N. Matthews, pp. 25-61. Altamira Press, Nueva York.

Castañeda, Q. y C.N. Matthews (eds.) 2008. Ethnographic Archaeologies: Reflections on Stakeholders and Archaeological Practices. Altamira Press, Nueva York.

Cox, A. 2005. What are communities of practice? A comparative review of four seminal works. Journal of Information Science 31(6):527-540.

Dilling, L. y M.C. Lemos 2011. Creating usable science: opportunities and constraints for climate knowledge use and their implications for science policy. Global Environmental Change 21:680-689.

Eckert, P. y S. McConnel-Ginet 1992. Think practically and look locally: language and gender as community-based practice. Annual Review of Anthropology 21:461-490.

Edgeworth, M. 2006. Multiple origins, development, and potential of ethnographies of archaeology. En Ethnographies of Archaeological Practice: Cultural Encounters, Material Transformations, editado por M. Edgeworth, pp. 1-19. Altamira Press, Nueva York.

Edgeworth, M. (ed.) 2006. Ethnographies of Archaeological Practice: Cultural Encounters, Material Transformations. Altamira Press, Nueva York.

Ferguson, J. 1994. The Anti-Politics Machine: "Development”, Depoliticization, and Bureaucratic Power in Lesotho. University of Minnesota Press, Minneapolis.

Gerunds, A. 2007. Grounding the Past: The Praxis of Participatory Archaeology in the Mixteca Alta, Oaxaca, Mexico. CNWS Publication, Leiden.

Glover, J.B., D. Rissolo y J. P. Mathews 2011. The hidden world of the maritime Maya: lost landscapes along the north coast of Quintana Roo, Mexico. En Maritime Cultural Landscapes, editado por B. Ford, pp. 195-216. Springer, Nueva York.
GTZ (Cooperación Técnica Alemana) 2011. Guía de Sistematización (1 septiembre 2011). http://www.gtz.de/en/ dokumente/sp-sl-guia-de-sistematizacion-documento-completo. pdf (1 julio 2011).

Gust, J. y J.P. Mathews 2011. Dyewood, sugar, rum, and piracy: the historic period of the Costa Escondida, Quintana Roo, Mexico. Ponencia presentada en el Annual Meeting of the American Anthropological Association, Montreal.

Hildreth, P. y C. Kimble 2004. Knowledge Networks: Innovation through Communities of Practice. Idea Group Inc., Hershey, Pennsylvania.

Hutson, S.R. 2010. Dwelling, Identity and the Maya: Relational Archaeology at Chunchucmil. Altamira Press, Nueva York.

Kanché 2010. Kanché Asociación Civil (1 septiembre 2011). www.kanche.org (1 marzo 2010).

Kretzmann, J. y J. McKnight 1993. Building Communities from the Inside Out: A Path Toward Finding and Mobilizing a Community's Assets. Institute for Policy Research, Evanston.

Kus, S. 2010. Observing the past, participating in the present: Archaeologically informed ethnography. The SAA Archaeological Record 10(1):13-16.

Lave, J. y E. Wenger 1991. Situated Learning: Legitimate Peripheral Participation. Cambridge University Press, Cambridge.

Leeuwis, C. y R. Pyburn (eds.) 2002. Wheelbarrows Full of Frogs: Social Learning in Rural Resource Management. Koninklijke van Gorcum, Assen.

Li, T.M. 2007. The Will to Improve: Governmentality, Development, and the Practice of Politics. Duke University Press, Durham.

Lynott, M.J. y A. Wylie (eds.) 2000. Ethics in American Archaeology: Challenges for the 1990s. 2nd edition. Society for American Archaeology, Washington, D.C.

Marshall, Y. 2002. What is community archaeology? World Archaeology 34:211-219.

McGuire, R.H. 2008. Archaeology as Political Action. University of California Press, Berkeley.

Mortensen, L. y J. Hollowell (eds.) 2009. Ethnographies and Archaeologies: Iterations of "Heritage" and the Archaeological Past. University Press of Florida, Gainesville.

Moser, S., D. Glazier, J.E. Phillips, L. Nasser el Nemr, M.S. Mousa, R.N. Aiesh, S. Richardson, A. Conner y M. Seymour 2002. Transforming archaeology through practice: strategies for collaborative archaeology and the community archaeology project at Quseir, Egypt. World Archaeology 34:220-248.

Puerta Verde 2010. Puerta Verde (1 septiembre 2011). www. puertaverde.com.mx (1 marzo 2010).

Reed, M.S. 2008. Stakeholder participation for environmental management: a literature review. Biological Conservation 141:2417-2431.

Rissolo, D. y J.P. Mathews 2005. Archaeologists working with the contemporary Yucatec Maya. En Lifeways in the Northern Maya Lowlands: New Approaches to Archaeology in the Yucatán 
Peninsula, editado por J.P. Mathews y B.A. Morrison, pp. 198209. University of Arizona Press, Tucson.

Sabloff, J.A. 1998. Distinguished lecture in archaeology: communication and the future of American archaeology. American Anthropologist 100:869-875.

Santoro, C.M, V.G. Standen, L.A. Romero G. y P. Ayala 2003. Editorial. Chungara Revista de Antropología Chilena 35:171-174.

Sullivan, P.R. 2004. Xuxub Must Die: The Lost Histories of a Murder on the Yucatan. University of Pittsburgh Press, Pittsburgh.

Tekelenburg, A., E. Goewie y A. Rojas 2002. A management toolkit for interactive learning: Farm innovation with Cactus pear and cochineal in Cochabamba. En Wheelbarrow Full of Frogs. Social Learning in Rural Resource Management, editado por C. Leeuwis y R. Pyburn, pp. 167-182. Koninklijke van Gorcum, Assen.
Walker, C.J. 2009. Heritage or Heresy? The Public Interpretation of Archaeology and Culture in the Maya Riviera. University of Alabama Press, Birmingham, Alabama.

World Wildlife Fund 2010. Mesoamerican Reef Projects - Yum Balam Flora and Fauna Protected Area (1 septiembre 2011). http:// www.worldwildlife.org/what/wherewework/mesoamericanreef/ projectspage-yumbalam.html (1 marzo 2010).

Wylie, A. 1995. Alternative histories: epistemic disunity and political integrity. En Making Alternative Histories, editado por P.R. Schmidt y T.C. Patterson, pp. 255-272. School of American Research Press, Santa Fe.

Yum Balam 2010. Yum Balam Área de Protección de Flora y Fauna (1 septiembre 2011). http://pyucatan.conanp.gob.mx/ yumbalam.htm (1 marzo 2011). 